\title{
Conversación sobre la obra de Sergio Galindo ${ }^{1}$
}

\section{Conversation on the Works of Sergio Galindo}

\author{
John Brushwood, Guadalupe Flores, \\ Valentina Pabello, Ángel José Fernández, \\ Sergio González Levet, Leticia Ramírez \\ y Renato Prada ${ }^{2}$ \\ Universidad Veracruzana
}

\section{RESUMEN}

En el diálogo que sostienen un investigador experimentado y los integrantes del Seminario de Semiótica, se ponen de relieve los recursos narrativos de Sergio Galindo: el estilo indirecto libre, el contraste entre la voz y la focalización, la sutil mezcla de narración extradiegética e intradiegética, las aperturas in media res-que dan paso a la recapitulación de los hechos precedentes-, el desencuentro entre la moral dominante y la moral desafiante -esta última siempre asumida por los protagonistas, víctimas al final, de los representantes de aquélla. Esos recursos, aseguran los dialogantes, son elementos que marcarán las investigaciones posteriores, cuando los planteamientos teóricos y críticos los hayan estudiado a profundidad. La vinculación docencia-investigación resulta ser una experiencia formativa que tiene como base una pasión compartida: la lectura de la narrativa del escritor veracruzano Sergio Galindo, pues más allá de las dubitaciones del origen teórico de este o aquel concepto, lo importante es incentivar y sostener el espíritu crítico en los nuevos lectores.

${ }^{1}$ Véase (1979, enero-junio). Conversación sobre la obra de Sergio Galindo. Semiosis (2), pp. 75-81. Xalapa, Universidad Veracruzana. Actualizado.

${ }^{2}$ El Seminario de Semiótica estuvo integrado por Guadalupe Flores, Valentina Pabello, Ángel José Fernández, Sergio González Levet, Leticia Ramírez y Renato Prada. 
Palabras clave: Textualidad; intertextualidad teatral; estilo indirecto libre; voces; puntos de vista.

\section{Abstract}

In the dialogue held by an experienced researcher and the members of the Semiotic Seminar, Sergio Galindo's narrative resources are highlighted: the free indirect style, the contrast between the voice and the focalization, the subtle mixture of extradiegetic and intradiegetic narration, the openings in media res -which give way the recapitulation to the preceding events-, the disagreement between the dominant morality and the challenging morality - the latter always assumed by the protagonists, victims in the end, of the former's representatives. Those resources, say the dialogue participants, are elements that will mark subsequent research, when theoretical and critical approaches have studied them in depth. The teaching-research link turns out to be a formative experience that is based on a shared passion: reading the narrative of Veracruz writer Sergio Galindo, because beyond the doubts of theoretical origin of this or that concept, the important thing is to encourage and sustain the critical spirit in new readers.

Key words: Textuality; theatrical intertextuality; free indirect style narrative; voices; point of view.

Brushwood. Conocí la obra de Galindo en un estado de inocencia, antes de interesarme en la semiótica, en el estructuralismo y en todo eso. De manera que nunca he analizado la obra de Sergio desde ese punto de vista. Últimamente, he pensado que sería interesante estudiar sus novelas, sobre todo con una idea de Genette, que es la división entre la voz narradora y el ojo o, como dice Genette, focalización. ¿Por qué me parece muy importante en la novela de Sergio esa diferencia? Sobre todo porque él emplea, siempre ha empleado en su narración, un cambio muy sutil de posiciones narrativas. Cuando emplea, por ejemplo, dentro de un solo párrafo, una posición exterior, emplea la tercera persona; y luego cambia, para ver desde un punto de vista de su personaje, pero siempre empleando la tercera persona, con la voz exterior, pero ya con el ojo 
interior, y termina en el mismo párrafo empleando la voz interior, una del personaje y el ojo, el focalizador, también del personaje. Ya que tiene esa característica de emplear las tres posiciones, tan rápidas, me parece que sería interesante estudiar todo eso de una manera más coherente.

Yo creo que es por eso que La comparsa funciona como novela. De otra manera, le sería imposible presentar a tanta gente en tantos episodios, algunos muy cortos, si no fuera por esa capacidad de cambiar la posición narrativa.

¿Qué están haciendo con Polvos de Arroz? Pues esa novela también me parece susceptible a esa clase de análisis. Porque supongo, sin haber releído la novela durante varios años, que se ve todo o casi todo desde el punto de vista Camerina, ¿no?

Seminario. Sí, es, más o menos, la dominante.

BRushwOOD. ¿Pero no es la voz de ella, verdad?

SEminario. Sí, eso es lo que hemos descubierto en él. Lo que nosotros decíamos es que no tenía la rigidez narrativa de mantener sólo una perspectiva, sino que inmediatamente se iba hacia la otra. BRushwOOD. ¿Recuerdan quién inventó el término estilo indirecto libre? ¿Fue Hansfret realmente? No sé... Yo creía que había sido un crítico español.

SEminario. En el problema del discurso, más bien fue el discípulo de Saussure. Él utilizó esto del discurso indirecto libre, que después se lo llamó estilo indirecto. Pero no sé si en la crítica, quizás, a su vez...

BRUSHWOOD. ¿Y en El hombre de los hongos? Realmente, no recuerdo; como les explicaba, no es mi novela predilecta, de manera que no la he estudiado mucho.

Seminario. En esa novela, Galindo trabaja en primero persona, pero, sin embargo, hay una dualidad: es el yo, Emma, la que cuenta, pero, a veces, el punto de vista de ella no predomina; hay veces predomina, hay veces no.

Brushwood. Esa voz que emplea, en primera persona, ¿está fuera o dentro de la novela?

SEminario. Es decir, ya estaría fuera, puesto que es una recapitulación; no es al mismo tiempo que el desarrollo de la diégesis. 
Brushwood. Eso es lo que me preguntaba; es más bien como la primera persona de la novela picaresca.

Seminario. Exacto. No es como en algunas novelas de Becket, en las cuales la voz se organiza en el momento que está hablando en el discurso. Aquí es siempre la recapitulación; ese sentido no presenta mayor dificultad tampoco.

BRUSHWOOD. Una primera persona de esa índole me parece muy poco distinta de una tercera persona.

SEminario. Está muy cerca de la neutralidad.

BRushwoOD. La tercera persona, al fin y al cabo, tiene que ser una primera persona. Siempre ha sido una equivocación la de decir que es una voz en primera persona; es una voz que emplea la tercera persona. Detrás de ese empleo de la tercera persona, tendrá que ser una primera persona.

SEMINARIO. Un narrador, que es el que afirma y organiza el discurso. Yo creo que eso es un poco lo que caracteriza a El hombre de los hongos. Sin embargo, hay una ambigüedad: no es tan diáfano el equilibrio entre yo y él, es decir, ese yo no podría ser neutralizado en un él, como ocurre en Camus: en él puede ser perfectamente neutralizado; en un éste no, porque hay una ambigüedad del juego. Yo creo que tiende hacia una lectura psicoanalítica, por la ambigüedad del juego de este hombre, el elemento simbólico en él.

Brushwood. No estudian Nudo, ¿ंverdad?

Seminario. No, Nudo no la hemos estudiado.

BRushwoOD. Hay cambios interesantes en Nudo, cambios de la voz narradora.

SEminario. En términos generales, sin tomar la obra como objeto de estudio y viendo toda la narrativa de Sergio, creo que a partir de Nudo hay un cambio en lo de estas técnicas: lo de la focalización, la confusión aparente de una primera y tercera personas dentro del discurso, por una parte; y la técnica teatral, muy empleada en las anteriores novelas, cambia radicalmente en Nudo. Es otra forma de enfocar la situación, aparte de que temáticamente se sale de la tradición del autor. Nudo me parece que innova otras situaciones: por ejemplo, mezcla ya una técnica teatral, en un capítulo que recuerdo; utiliza la analepsis, en otro capítulo, que no la utilizaba 
muy comúnmente. Me parece que eso hace de ella una novela muy diferente a las otras. Está hecha a base de recuerdos. Inclusive, hay un capítulo de la infancia de los personajes y después, hacia el final de la novela, hay otra intervención de un dialogo teatral... Todo esto me parece que es interesante dentro de la concepción de la obra en general.

BrushwOOD. ¿Le parece que esa analepsis que emplea en Nudo es distinta de lo que ha hecho en El bordo cuando, en cierto momento de una escena, de pronto, el personaje está pensando efectivamente, viviendo a base una época pasada, no en forma de flash-back, sino más bien en el campo de referencia... Pero yo creo que lo que usted indica realmente es distinto de lo que estoy describiendo yo; no estoy seguro, me parece distinto.

Seminario. Yo creo que sí es distinto, porque en El bordo hace ese recuerdo, mientras que en Nudo no se recuerda solamente, sino que se está viviendo esa historia o pasado; esa analepsis empieza a vivirse, y en el contexto general de la obra, ya que está dada la situación, cosa que no ocurre en El bordo: en éste, está realmente recordando y añorando una situación pasada. Ahora, la impresión que a mí me dio, así, a una primera lectura, porque no la he trabajado, es que es muy desarrollada técnicamente. Se ve una labor técnica que no se ve en los otros.

BRushwOOD. Yo creo que el autor está absolutamente consciente de eso; él reconoce a esa novela como mucho más complicada, técnicamente, que las otras. De manera que uno pudiera decir que hay cierto mensaje en las novelas de Galindo, hasta Nudo, que luego cambia, porque el mensaje en Nudo me parece mucho más vinculado a la manera de expresarse o a la técnica de narrar, porque sin esta técnica no puedo decir cuál es el mensaje de El Bordo, o de La comparsa, o de Polvos de arrož, no puedo señalar el mensaje de Nudo sin hablar de la técnica; y yo creo que hay diferencia; y no sé si en el caso de El hombre de los hongos será igual.

SEMINARIO. No, me parece que pertenece, más a bien, a un clima anterior; está escrita antes ¿no? La primera versión de El hombre de los hongos está escrita antes... 
BRushwoOd. Yo creo que hacía años que estaba jugando con esa idea. Yo creo que no la escribió mucho antes, sino que había pensado la novela antes; sino que había pensado la novela muchas veces antes de escribir; y probablemente no la hubiera escrito sin la promoción de parte de Emilio Carballido.

SEMINARIO. ¿Por qué no nos habla de la técnica teatral? Yo recuerdo un artículo de usted, publicado en la Revista de Bellas Artes, hablando particularmente de La comparsa, donde planteaba esta técnica teatral que nunca he tenido clara.

BRushwoOD. Sí, recuerdo bien ese ensayo al que usted se refiere: se habla del cambio de la voz. Pero eso ocurre en todas las novelas, nada más que en La comparsa me parece de importancia fundamental; por eso, episodios tan cortos, que si no pudieran desarrollar y cambiar el punto de vista tan rápido no habría manera de conocer a esa gente. Cuáles son las oportunidades que tenemos, en 160 páginas, más o menos, de conocer a Arnold Huerta, a toda esa gente que entra y sale; es algo como un teatro, pero nada más: como una especie de revista, no como una comedia.

SEminario. Esta técnica teatral está más bien analizándose desde fuera de la novela; o..., es lo que no entiendo, quizá ese matiz... BRushwood. Bueno, estoy tratando de recordar, francamente, porque, por lo que recuerdo yo, es lo del cambio de la voz, hablar de técnica teatral... realmente no recuerdo lo que dije específicamente. Pero seguramente, en el análisis del texto yo estaba afuera del texto, observando un fair accompli, que es una cosa completamente distinta de los que se hacen con Barthes cuando se estudia la estructuración del texto. Yo estaba seguramente afuera.

SEminario. Lo que recuerdo, porque también leí hace mucho tiempo ese texto, era que esa técnica teatral se estaba convirtiendo en un "estilo" de Sergio Galindo, porque desde la primera situación, desde el inicio del discurso, ya se empezaban a plantear las situaciones de la misma manera que se plantea técnicamente una situación para el teatro. Que todo está dado desde un principio.

SEminario. Ahora, lo que quizás anoche decía acerca de esto de la repetición, quizá sí. 
BRushwoOd. Bueno, eso tiene que ver con lo que decía esta mañana de Brox y Wild y la entrada en la histoire, lo que yo llamo historia. Ellos hablan de la materia anecdótica, que para empezar el autor tiene que escoger un momento en esa historia, en esa materia para entrar; y Sergio entra en un momento culminante, cuando algo muy importante está pasando; y luego tiene que dar muy rápidamente todos los detalles, todos los pormenores necesarios. Eso es lo que pasa en el teatro con frecuencia, que empezamos en un momento, el telón se levanta, luego entra una persona o vemos a un personaje que nos dice algo importante en un momento de crisis, algo que está pasando, y luego el primer acto consta de una justificación de ese momento; luego en el segundo acto, con frecuencia, experimentamos la analepsis para entender lo que pasó o las circunstancias que causó el primer acto; y el tercer acto es el desenvolvimiento. Pero creo que tenía que ver con ese momento de entrada. Por ejemplo, La comparsa, en que empezamos o vemos el primer momento de Bartolomé; en El bordo, entramos en el momento cuando Hugo y Esther están llegando a la casa; de manera que entramos inmediatamente, de una manera muy abrupta.

Seminario. Bueno, eso sucedería también, tal vez, en Polvos de arroz: inmediatamente, se va al conflicto de Camerina; después se va al pasado, porque ella llega a México y todo eso.

Brushwood. Entonces, lo que decíamos de la repetición, si el narrador entra de esa manera en la materia, en general plantea, casi inmediatamente, el conflicto que va a desarrollarse; y luego, a veces, se desarrolla el conflicto. Pero a veces, nada más va repitiendo el conflicto en la serie de acontecimientos, que parecen acontecimientos distintos, pero que, en el fondo, llevan un mismo mensaje o la misma significación. Eso es interesante, porque, a veces, me parece que los acontecimientos aumentan la tensión poco a poco, con cada acontecimiento un poco más intenso que el anterior. En otras, me parece que mantienen más o menos el mismo nivel de tensión, pero que la totalidad de la serie nos da la impresión de un conflicto llegado a su máximo desarrollo. Pero es una repetición escondida, si el narrador lo hace bien, porque leemos los acontecimientos realmente sin pensar que es una repetición; nada 
más que nosotros, que hacemos análisis, vemos la repetición y la vemos; apreciamos, desde un principio, el conflicto; posiblemente, después de leer la novela, que es una lectura muy distinta de la lectura nuestra, en que buscamos el conflicto. Por eso, hay dos maneras de leer, que son casi contrarias. Efectivamente -y eso a mí me ha pasado, no sé si con frecuencia, pero por lo menos de vez en cuando-, leo la novela y casi al final de la novela descubro lo que hubiera descubierto al principio; y luego, si no releo la novela, por lo menos la repienso; y es casi como otra lectura: vuelvo al principio y experimento todo de nuevo.

Seminario. Otro problema que se plantea en Polvos de arroz es recordar lo que se llama la moral textual y la moral extratextual; y el conflicto ente ambas. Eso me parece casi un leit-motiv en él: hay un personaje que quiere establecer una moral que está fuera de la moral dominante. Emma también muere; el hombre de los hongos se va; y todo queda en la destrucción total. En La comparsa, Clementina Pereda es descubierta realmente sin máscara y sufre las consecuencias de la moral dominante.

BRushwood. Bueno, me parece absurdo hablar de la obra de Galindo como una novela de denuncia, sino más bien de descubrimiento. Como es un autor muy profundo, yo creo que descubre nuevas maneras de manifestación y ve el conflicto posible con la moral dominante. Si bien él no toma partido, tampoco; mientras que el que denuncia toma partido. Galindo se aleja del conflicto y sólo deja los elementos para que el lector los analice... Me estoy acordando de un cuento que se llama "Me esperan en Egipto", donde Rodrigo Mier, que es un bibliotecario que va a conseguir con sus ahorros un viaje, se convierte en una víctima del oportunismo de otras gentes y finalmente no consigue lo que toda su vida trató de conseguir y se aísla hasta morir... 